\title{
Wideband beamspace squint user grouping algorithm based on subarray collaboration
}

\author{
Xiaoliang $\operatorname{Pan}^{*}{ }^{\mathbb{0}}$, Chunguo Li and Luxi Yang
}

\section{${ }^{*}$ Correspondence:}

xlpan@seu.edu.cn

School of Information

Science and Engineering,

Southeast University,

Nanjing 210096, China

\begin{abstract}
The beam direction constrained problem is one of the important issues to be solved in millimeter-wave (mmWave) wideband communications when serving multi-user with squint beams whose direction varies with frequency. In this paper, we improve the number of simultaneous users served by collaboratively transmitting squint beams among multi-subarray at the base station (BS) end in a downlink multi-user line-ofsight (LoS) scenario, and reduce the interference among co-channel squint beams by a beam domain approach. The optimization problem of maximizing the number of users served in the system by transmitting beams in the two-dimensional beamspace of the planar antenna subarray is proposed and its suboptimal algorithm is given. Finally, the feasibility of the proposed method and the performance of the proposed algorithm are verified by numerical simulations.
\end{abstract}

Keywords: Millimeter-wave, Wideband array, Squint multi-beam, Beamspace

\section{Introduction}

Multi-beam antenna technologies have received extensive attention in 5G wireless communications [1-3], where systems based on hybrid digital-analog antenna array structures have been widely studied for their maximum sum rate performance in sparsely scattered millimeter-wave (mmWave) wireless communication scenarios that are closer to the capacity of the channel, while using only a small number of radio frequency (RF) links [4]. Since the available spectrum resources in the mmWave band cover a very wide range, when the system operates with a very wide bandwidth, such an antenna array with a common set of ideal phase shifters for all sub-bands in the analog domain will inevitably experience a shift in the beam direction between different frequencies [5, 6]. Capitalizing on this phenomenon, several user equipments (UEs) are served in the literature [1] by using squint multi-beam at the base station (BS) end. Where the so-called squint multi-beam is the multiple steering beams whose main direction changes with frequency over a wide frequency range via a group of non-ideal time delayers in the RF domain, such as the ideal phase shifter group. As the phase of the ideal phase shifter remains the same value in the operating frequency range, the array response of the wideband phased array beam using the ideal phase shifter group remains constant over the corresponding frequency range. Consequently its can further reduce the number of RF

(c) The Author(s) 2022. Open Access This article is licensed under a Creative Commons Attribution 4.0 International License, which permits use, sharing, adaptation, distribution and reproduction in any medium or format, as long as you give appropriate credit to the original author(s) and the source, provide a link to the Creative Commons licence, and indicate if changes were made. The images or other third party material in this article are included in the article's Creative Commons licence, unless indicated otherwise in a credit line to the material. If material is not included in the article's Creative Commons licence and your intended use is not permitted by statutory regulation or exceeds the permitted use, you will need to obtain permission directly from the copyright holder. To view a copy of this licence, visit http:// creativecommons.org/licenses/by/4.0/. 
links required for the antenna array while maintaining the multi-beam nature of the array in wideband scenarios.

However, in the literature [6], it was found that the main direction of the squint beam is constrained after the array response of the wideband array is determined. When the planar array employs a group of ideal phase shifters in the analog domain, it behaves as if the squint beam and the main beam at the center frequency need to maintain the same transmit horizontal azimuth for a certain coordinate system, which greatly limits the application of squint multi-beam in mmWave wideband wireless communications. As the solutions, one of them is to study the circumvention of this squint beam angle limitation problem, and in [7], by using a wideband analog linear array at the BS end, it is possible to cover user paths with different horizontal azimuth angles simultaneously through several donut-shaped squint main beams in the 3D space. The other is that this problem can be mitigated by the cooperative efforts of multiple BS ends in the communication network, as well as the collaboration of several subarrays at one BS end. In this paper, we provide multi-subarray at the BS end and multiplex the array responses of these subarrays to cover more angular space by the squint beams as one of the solutions to alleviate this problem.

Nevertheless, there is a possibility of co-channel beams across multi-subarray, which can cause inter-beam interference problems. In contrast, the beamspace domain, due to the orthogonality between multiple beams in its space, is used as beamspace channel representation [8] on the one hand, and can be used as the codebook for the actual beams at the transmitter and receiver sides through the analog domain phase shifter set on the other hand. In this paper, we ensure the orthogonality of the same frequency beams between subarrays by selecting vectors in the beamspace as the response vectors of the subarrays transmitting beams. Moreover, we find that this orthogonal beam is compatible with the uniformly quantized phase shifter group, namely, the quantized phase shifter group can be used to realize the orthogonal beam in the transmit beamspace domain [9]. In addition, the approach proposed in this paper has the opportunity to be combined with existing techniques [10-14] to further improve the performance of Internet of Vehicles (IoV) systems.

The contributions of the paper are summarized as follows.

- We propose an approach to partition the beamspace based on the maximum elevation angle of the main beam at the center frequency, and give the selection of the corresponding orthogonal beams in the beamspace.

- We show the relationship between the beamspace and the phase difference of adjacent elements of the antenna array, and verify that it is compatible with antenna arrays using uniformly quantized phase shifter sets by transmitting beams through beamspace codebooks.

- We propose a squint user grouping optimization problem based on the beamspace codebook to maximize the number of users served in the system, and we provide a suboptimal algorithmic implementation of it.

The rest of this work is organized as follows. In Sect. 2, we first give the system model, followed by the way of beamspace partitioning for a given maximum main beam 


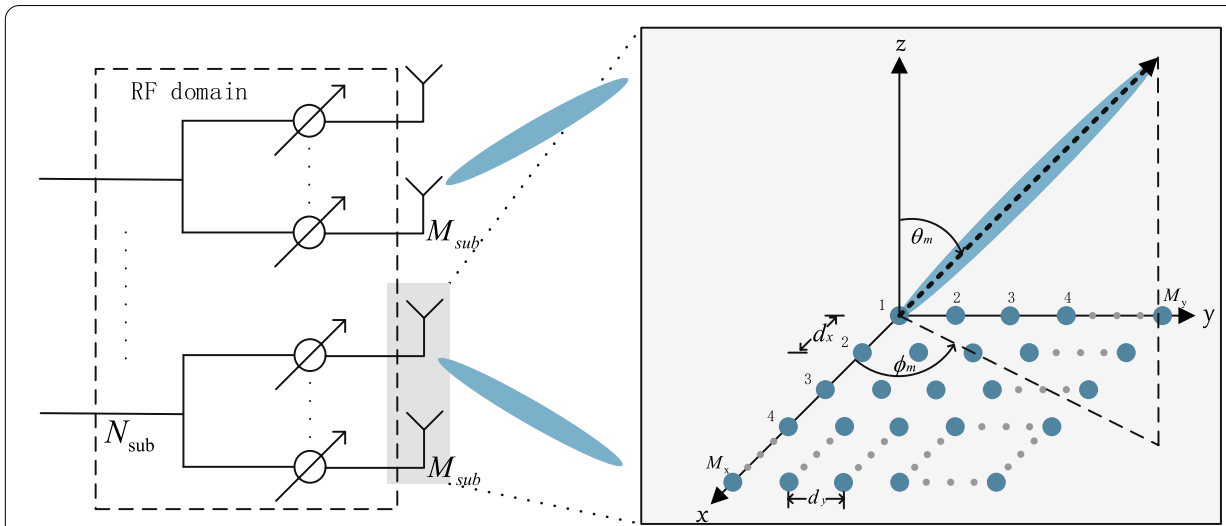

Fig. 1 The array structure of multiple wideband phased arrays at the BS side, and the coordinate system of each subarray

elevation angle, and finally discuss the effect of phase shifter quantization on the beamspace codebook. In Sect. 3, we start with a single subarray squint grouping optimization problem, followed by a collaborative user grouping optimization problem for multisubarray based on the beamspace codebook, and suggest a suboptimal algorithm for it. In Sect. 4, simulation results are conducted to verify the feasibility of the proposed grouping method and the performance of the proposed algorithm, before concluding the paper in Sect. 5.

Notation: Boldface uppercase letters, boldface lowercase letters, and lowercase letters are used to denote matrices, vectors and scalars, respectively; The superscripts $(\cdot)^{T}$ and $(\cdot)^{H}$ denote the transpose and conjugate transpose, respectively; $|\cdot|$ is the amplitude of a complex value; $\lfloor\cdot\rfloor$ and $\lceil\cdot\rceil$ denote floor and ceiling function, respectively; $\|\cdot\|_{0}$ represents the number of non-zero elements function of a matrix; $\operatorname{card}(\cdot)$ denotes the cardinal number of a set.

\section{Beamspace of arrays with restricted elevation beam angle}

In this section, the system model will be presented at first, followed by the investigation of the available codebook in the beamspace when the maximum elevation angle of the transmitting beam is limited, and finally, the effect of multi-subarray on the orthogonality of the beam domain vectors and the matching problem between the phase shifter quantization and the beam domain codebook will be discussed.

\subsection{System model}

We assume a single-cell downlink topology containing one BS and a number of UEs, where the BS side consists of multi-subarray and the UE end is all equipped with a single antenna. As shown in Fig. 1, all subarrays at the BS end are composed of wideband phased planar arrays of the same size, with the set of subarrays as $\mathcal{N}_{\text {sub }}=\left\{1,2, \ldots, N_{\text {sub }}\right\}$, and each subarray consists of a rectangular antenna array with the elements number of $M_{\text {sub }}=M_{x} \times M_{y}$, whose spacing between adjacent elements is one half of the wavelength corresponding to the center working frequency. The set of UEs in the system is $\mathcal{N}_{u}=\left\{1,2, \ldots, N_{u}\right\}$. Considering the long distance between the transceiver and the small size of the mmWave subarray used, it is assumed that the difference in 
angle-of-departure (AoD) of the same channel path between different subarrays can be neglected. Without loss of generality, the horizontal angle $\phi_{m}$ and the elevation angle $\theta_{m}$ of the steering beam emitted by subarrays are all within the interval $[0, \pi / 2]$.

In a downlink LoS scenario where each subarray at the BS end serves the UE individually, the equivalent baseband received signal of its $i$-th UE $\left(i \in \mathcal{N}_{u}\right)$ can be expressed as

$$
r_{i}=\underbrace{\sqrt{\rho_{i}} \mathbf{h}_{i}^{H} \mathbf{b}_{i} s_{i}}_{\text {signal }}+\underbrace{\sum_{l \in \mathcal{N}_{u}^{i}} \sqrt{\rho_{l}} \mathbf{h}_{i}^{H} \mathbf{b}_{l} s_{l}}_{\text {interference }}+\omega_{i},
$$

where $\rho_{i}$ is the power of transmitted steering beam for this UE, $\mathbf{h}_{i}$ is its channel vector when the subarray serves this $\mathrm{UE}, \mathbf{b}_{i}$ is the precoding vector of this subarray, and $s_{i}$ is the user signal which obeys the $\mathcal{C N}(0,1)$ distribution. $\mathcal{N}_{u}^{i}$ is the set of co-channel UEs of this UE in the system, which satisfies $\lambda_{l}=\lambda_{i}$ when $l \in \mathcal{N}_{u}^{i}$, and $\lambda_{i}$ denotes the working wavelength of this UE. $\omega_{i} \sim \mathcal{C N}\left(0, \sigma_{i}^{2}\right)$ is the noise at this UE end.

$$
\mathbf{h}_{i}=\alpha_{i} \sqrt{M_{\mathrm{sub}}} \mathbf{a}\left(\phi_{i}, \theta_{i}, \lambda_{i}\right),
$$

where $\mathbf{a}\left(\phi_{i}, \theta_{i}, \lambda_{i}\right)$ is the normalized array response ${ }^{1}$ at the transmitting subarray end, $\alpha_{i}=\left|\alpha_{i}\right| e^{j \psi_{i}}$ is the channel coefficient, and according to the Friis transmission formula $[15,16]$, we have

$$
\left|\alpha_{i}\right|=\frac{\lambda_{i} \sqrt{D_{0}}}{4 \pi d_{i}}
$$

where $d_{i}$ is the distance between this UE end and the BS side, and $D_{0}$ is the directivity of the array factor, which can be approximated as [17]

$$
D_{0} \approx \frac{\pi^{2}}{\Theta_{h}^{i} \Psi_{h}^{i}}
$$

where $\Theta_{h}^{i}, \Psi_{h}^{i}$ are the elevation and horizontal orientation half-power beam width (HPBW) of the transmitting main beam, respectively, and according to (6-98) and (6-99) in [17], we get

$$
\begin{aligned}
& \Theta_{h}^{i}=1 / \sqrt{\cos ^{2} \theta_{i}\left[\Theta_{x 0}^{-2} \cos ^{2} \phi_{i}+\Theta_{y 0}^{-2} \sin ^{2} \phi_{i}\right]}, \\
& \Psi_{h}^{i}=1 / \sqrt{\Theta_{x 0}^{-2} \sin ^{2} \phi_{i}+\Theta_{y 0}^{-2} \cos ^{2} \phi_{i}},
\end{aligned}
$$

where $\Theta_{x 0} \approx 2 \arcsin \left(\frac{2.782}{\pi M_{x}}\right)$ and $\Theta_{y 0} \approx 2 \arcsin \left(\frac{2.782}{\pi M_{y}}\right)$.

Following (1), the signal-to-noise ratio of this UE can be obtained as

\footnotetext{
${ }^{1}$ The array response can also be expressed by the phase difference of adjacent elements as a parameter, in this case the wavenumber in (9) and (10) is $k=2 \pi / \lambda_{i}$.
} 


$$
\operatorname{SINR}_{i}=\frac{\rho_{i}\left|\mathbf{h}_{i}^{H} \mathbf{b}_{i}\right|^{2}}{\sum_{l \in \mathcal{N}_{u}^{i}} \rho_{l}\left|\mathbf{h}_{i}^{H} \mathbf{b}_{l}\right|^{2}+\sigma_{i}^{2}} .
$$

Thus the sum rate of the system can be written as

$$
R=\sum_{i \in \mathcal{N}_{u}^{\prime}} \log _{2}\left(1+\mathrm{SINR}_{i}\right) \quad \text { bits } / \mathrm{s} / \mathrm{Hz}
$$

where $\mathcal{N}_{u}^{\prime}$ is the set of users served simultaneously.

\subsection{Beamspace on center frequency}

When considering the LoS scenario, the beam domain is divided into $M_{x} \times M_{y}$ small zones, each corresponding to an orthogonal beam in the beamspace, so the BS can distinguish the UEs in different small zones by the orthogonal beams of the beamspace [9]. We take the set of orthogonal beams in the beamspace as the codebook of the precoding vector, and when the AoD of a UE appears in a certain zone, the vector corresponding to that zone in the beamspace is used as the transmitting precoding vector to ensure the orthogonality among multiple transmitting beams.

From the perspective of the phase shifter group [6, 17] the so-called beamspace [9], that is, the DFT matrix column space formed by a set of orthogonal steering vectors constructed by taking values of uniform quantization points in the range of one phase period $[0,2 \pi)$ as the phase difference $\beta$ of adjacent elements in the array. For a uniformly spaced linear array consisting of $M$ elements, let $\beta^{i}=2 \pi i / M, i \in\{0,1, \ldots, M-1\}$ such that $\mathbf{a}\left(\beta^{i}\right)^{H} \mathbf{a}\left(\beta^{j}\right)=0, \forall i \neq j$, where $\mathbf{a}(\beta, M)=\frac{1}{\sqrt{M}}\left[1, e^{-j \beta}, \ldots, e^{-j(M-1) \beta}\right]^{T}$ is the steering vector. And for a $M_{x} \times M_{y}$-dimensional rectangular planar array, with one of its vertices as the origin and making the $x$-axis and $y$-axis extend through the two sides of the antenna array respectively to establish the coordinate system, its steering vector may be expressed as $\mathbf{a}\left(\beta_{x}, \beta_{y}\right)=\mathbf{a}\left(\beta_{x}, M_{x}\right) \otimes \mathbf{a}\left(\beta_{y}, M_{y}\right)$ [15], where $\beta_{x}$ and $\beta_{y}$ are the emission phase difference between adjacent elements along the $x$-axis and $y$-axis of the antenna array, respectively. By the nature of the matrix Kronecker product $(\mathbf{A} \otimes \mathbf{B})(\mathbf{C} \otimes \mathbf{D})=(\mathbf{A C}) \otimes(\mathbf{B D}), \quad$ we $\quad$ have $\left(\mathbf{a}\left(\beta_{x}^{i}, M_{x}\right) \otimes \mathbf{a}\left(\beta_{y}^{l}, M_{y}\right)\right)^{H}\left(\mathbf{a}\left(\beta_{x}^{j}, M_{x}\right) \otimes \mathbf{a}\left(\beta_{y}^{k}, M_{y}\right)\right)=\left(\mathbf{a}\left(\beta_{x}^{i}, M_{x}\right)^{H} \mathbf{a}\left(\beta_{x}^{j}, M_{x}\right)\right) \otimes\left(\mathbf{a}\left(\beta_{y}^{l}, M_{y}\right)^{H} \mathbf{a}\left(\beta_{y}^{k}, M_{y}\right)\right)=0, \quad \forall i \neq j$ or $l \neq k$, which means the beamspace of the two-dimensional planar array can be constructed by following the way of the steering vector in the beamspace of the linear array.

As shown in Fig. 1, the phase difference [17] between its adjacent array elements along the $x$-axis and $y$-axis can be obtained when the transmitting main beam direction of a subarray is $\left(\phi_{m}, \theta_{m}\right)$, namely

$$
\begin{aligned}
& \beta_{x}=k d_{x} \cos \phi_{m} \sin \theta_{m}, \\
& \beta_{y}=k d_{y} \sin \phi_{m} \sin \theta_{m} .
\end{aligned}
$$

It can be seen that (9) and (10) also establish the connection between the beamspace and the main beam direction for the planar array.

Consider the main beam in the right half of Fig. 1 which is active only in the first quadrant, with $\phi_{m}, \theta_{m} \in[0, \pi / 2]$ in this case. Assume that the elevation angle $\theta_{m}$ of the main 
beam at the center frequency is chosen in the interval $\left[0, \theta_{m}^{\max }\right]$ and the elevation angle in the range $[0, \pi / 2]$ can be covered by the chosen frequency. Substituting $\theta_{m}^{\max }$ into (9) and (10) yields the corresponding $\beta_{x}$ and $\beta_{y}$ selection ranges of both $\left[0, \pi \sin \theta_{m}^{\max }\right]$, the corresponding set of available vectors in this beamspace is

$$
\begin{aligned}
& \mathbb{B}=\left\{\mathbf{b}_{\operatorname{card}\left(\mathcal{M}_{y}\right) i+l}=\mathbf{a}\left(\beta_{x}^{i}, M_{x}\right) \otimes \mathbf{a}\left(\beta_{y}^{l}, M_{y}\right):\right. \\
& \beta_{x}^{i}+\beta_{y}^{l} \leq \sqrt{2} \pi \sin \theta_{m}^{\max }, \theta_{m}^{\operatorname{card}\left(\mathcal{M}_{y}\right) i+l} \leq \theta_{m}^{\max }, \\
& \left.\beta_{x}^{i}=2 i \pi / M_{x}, \beta_{y}^{l}=2 l \pi / M_{y}, i \in \mathcal{M}_{x}, l \in \mathcal{M}_{y}\right\}
\end{aligned}
$$

where $\quad \mathcal{M}_{x}=\left\{0,1, \ldots,\left\lfloor\frac{\pi \sin \theta_{m}^{\max }}{2 \pi / M_{x}}\right\rfloor\right\}$ and $\mathcal{M}_{y}=\left\{0,1, \ldots,\left\lfloor\frac{\pi \sin \theta_{m}^{\max }}{2 \pi / M_{y}}\right\rfloor\right\} . \quad$ When $\operatorname{card}\left(\mathcal{M}_{x}\right)>1$, the phase difference of adjacent elements along the $x$-axis can be divided as

$$
\begin{cases}{\left[0, \pi / M_{x}\right),} & i=0, \\ {\left[\frac{2 \pi}{M_{x}}\left(\left\lfloor\frac{M_{x}}{2}\right\rfloor-0.5\right), \pi \sin \theta_{m}^{\max }\right],} & i=\left\lfloor\frac{\pi \sin \theta_{m}^{\max }}{2 \pi / M_{x}}\right\rfloor, \\ {\left[\frac{2 \pi}{M_{x}}(i-0.5), \frac{2 \pi}{M_{x}}(i+0.5)\right), i \in} & \left\{1, \ldots,\left\lfloor\frac{\pi \sin \theta_{m}^{\max }}{2 \pi / M_{x}}\right\rfloor-1\right\} .\end{cases}
$$

Similarly, the phase difference division of adjacent array elements along the $y$-axis can be obtained by rewriting $M_{x}$ in (12) as $M_{y}$, namely

$$
\begin{cases}{\left[0, \pi / M_{y}\right),} & l=0, \\ {\left[\frac{2 \pi}{M_{y}}\left(\left\lfloor\frac{M_{y}}{2}\right\rfloor-0.5\right), \pi \sin \theta_{m}^{\max }\right],} & l=\left\lfloor\frac{\pi \sin \theta_{m}^{\max }}{2 \pi / M_{y}}\right\rfloor, \\ {\left[\frac{2 \pi}{M_{y}}(l-0.5), \frac{2 \pi}{M_{y}}(l+0.5)\right), l \in} & \left.\left\{1, \ldots, \mid \frac{\pi \sin \theta_{m}^{\max }}{2 \pi / M_{y}}\right\rfloor-1\right\} .\end{cases}
$$

(12) and (13) jointly present an interval division of the two-dimensional continuous beam domain. In addition, $\beta_{x}^{i}+\beta_{y}^{l} \leq \sqrt{2} \pi \sin \theta_{m}^{\max }$ and $\theta_{m}^{\operatorname{card}\left(\mathcal{M}_{y}\right) i+l} \leq \theta_{m}^{\max }$ in (11) are the restrictions on the effective range of the beam interval obtained by summing up (9) and (10) as well as the maximum elevation angle of the main beam at the center frequency, respectively. The AoD of the actual LoS channel path is mapped to a certain zone in the beam domain by (12) and (13), and the codebook corresponding to this zone in (11) is taken as the precoding vector, which is the beamspace codebook-based precoding method we adopt.

\subsection{The effect of subarray on orthogonality in beamspace}

Assuming that the adjacent elements of a planar array composed of multi-subarray all maintain a half-wavelength spacing corresponding to the center frequency, in order to investigate the orthogonality of the beams between the subarrays, from the perspective of a large array it may be useful to rewrite the steering beam vector of the $k$-th subarray as

$$
\mathbf{a}_{k}\left(\beta_{x}^{i}, \beta_{y}^{j}\right)=[\underbrace{0, \ldots, 0}_{M_{\mathrm{sub}} \times(k-1)}, \mathbf{a}\left(\beta_{x}^{i}, \beta_{y}^{j}\right)^{T}, \underbrace{0, \ldots, 0}_{M_{\mathrm{sub}} \times\left(N_{\mathrm{sub}}-k\right)}]^{T} .
$$

Then the following two equations are valid, namely 


$$
\begin{aligned}
& \mathbf{a}_{k}\left(\beta_{x}^{i}, \beta_{y}^{j}\right)^{H} \mathbf{a}_{s}\left(\beta_{x}^{m}, \beta_{y}^{n}\right)=0, \\
& k, s \in \mathcal{N}_{\text {sub }} \text { and } k \neq s, \\
& i, m \in \mathcal{M}_{x}, \\
& j, n \in \mathcal{M}_{y},
\end{aligned}
$$

and

$$
\begin{aligned}
& \mathbf{a}_{k}\left(\beta_{x}^{i}, \beta_{y}^{j}\right)^{H} \mathbf{a}_{k}\left(\beta_{x}^{m}, \beta_{y}^{n}\right)=0, \\
& k \in \mathcal{N}_{\text {sub }}, \\
& i, m \in \mathcal{M}_{x}, \\
& j, n \in \mathcal{M}_{y} \\
& i \neq j \text { or } m \neq n .
\end{aligned}
$$

When each subarray precoding vector is selected from the beamspace shown in (11), (15) guarantees the orthogonality of the transmitted beams between subarrays, (16) guarantees the orthogonality between different beam vectors of the same subarray. Therefore, the orthogonality of the precoding vectors between subarrays and the orthogonality of multiple transmitted beams within the same subarray can be guaranteed when the same beamspace codebook is used for the precoding vectors of all subarrays.

\subsection{The effect of phase shifter quantization on beamspace}

For a linear array, the matrix composed of the phase shift vectors in its beamspace is actually a DFT matrix, so all the elements in it can be written in the form of a rotation factor power. And when all phase shifters in the phase shifter group of the antenna array are $Q$ bits uniformly quantized, let its individual quantized phase shift cells and the rotation factor have the same phase shift value as $\beta_{\text {cell }}=2 \pi / M$, so according to the steering vector formula of the linear array and $\beta^{i}=i \beta_{\text {cell, }}$, when satisfying $2^{Q} \beta_{\text {cell }} \geq(M-1) \beta^{(M-1)}$, that is, $2^{Q} \geq(M-1)^{2}$, all the beams in the beamspace codebook can be emitted, at which time the minimum number of quantized bits is $Q_{\min }=2\left\lceil\log _{2}(M-1)\right\rceil$.

For a planar array whose phase shifter group also adopts the above quantization, according to Eq. (37) in [6], it needs to satisfy $\left(M_{x}-1\right) \beta_{x}^{i}+\left(M_{y}-1\right) \beta_{y}^{l} \leq 2^{Q} \beta_{\text {cell }}$ when transmitting the beam through the beamspace in (11), therefore leading to

$$
Q_{\min }=\left\lceil\log _{2}\left[\left(M_{x}-1\right)\left(\operatorname{card}\left(\mathcal{M}_{x}\right)-1\right)+\left(M_{y}-1\right)\left(\operatorname{card}\left(\mathcal{M}_{y}\right)-1\right)\right]\right\rceil .
$$

Since the steering beams in the beamspace matrix can all be represented by a group of uniformly quantized phase shifters, we can realize the beamspace vector of the antenna array when the phase shifter group is unquantized by a uniformly quantized phase shifter group and its maximum transmit elevation angle is no greater than $\theta_{m}^{\max }$ once (17) is satisfied.

\section{User grouping based on collaborative subarrays of squint beams}

In this section, we will first discuss the interval partitioning problem of squint beams at vertical angles with a subarray as an example, and apply this partitioning method to the optimization problem of maximizing the total number of users served by multi-subarray, 
after which we will give a suboptimal algorithm implementation of this optimization problem.

\subsection{Squint user grouping method based on a single subarray}

When this planar subarray transmits steering beams, once the main direction $\left(\phi_{i}, \theta_{i}\right)$ of the steering beam at its center frequency is determined, the horizontal azimuth of its squint beam remains constant [6]. For better utilizing squint beams, the angular division grouping approach [7] can be performed according to the elevation HPBW $\Theta_{h}^{i}$ at the center frequency in the transmitting vertical azimuthal range $[0, \pi / 2]$. Assuming that $\left(\phi_{i}, \theta_{i}\right)$ is the center of the grouping Zone $i 0$, and the other groupings are divided into intervals according to Zone $i 0$ increasing or decreasing $\theta_{h}^{i}$ (except for the edge groupings), this squint grouping method can be expressed as

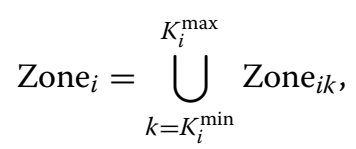

where $K_{i}^{\min }=-\left\lceil\frac{\theta_{i}}{\Theta_{h}^{i}}\right\rceil, K_{i}^{\max }=\left\lfloor\frac{\pi / 2-\theta_{i}}{\Theta_{h}^{i}}\right\rfloor$,

$$
\text { Zone }_{i k}= \begin{cases}{\left[0, \theta_{i}+(k+0.5) \Theta_{h}^{i}\right),} & k=K_{i}^{\min }, \\ {\left[\theta_{i}+(k-0.5) \Theta_{h}^{i}, \theta_{i}+(k+0.5) \Theta_{h}^{i}\right),} & k \in \mathcal{K}_{i}^{i}, \\ {\left[\theta_{i}+(k-0.5) \Theta_{h}^{i}, \pi / 2\right],} & k=K_{i}^{\max },\end{cases}
$$

where $\mathcal{K}_{i}=\left\{K_{i}^{\min }+1, K_{i}^{\min }+2, \ldots, K_{i}^{\max }-1\right\}$.

Equations (18) and (19) denote the group division of the elevation space $[0, \pi / 2]$ corresponding to the $i$-th subarray when its transmitting main beam elevation angle is $\theta_{i}, i \in \mathcal{N}_{\text {sub }}$. Assume that each group Zone ${ }_{i k}$ in (19) can have at most one transmitting squint beam, and each squint beam serves one UE whose horizontal azimuth AoD of its channel path is within the HPBW of the beam corresponding to the center frequency. After the horizontal azimuth angle $\phi_{i}$ of the transmitting steering beam of the subarray is determined, the number of users that can be served simultaneously by the subarray is further increased by adjusting the horizontal azimuth angle $\theta_{i}$ to change its angular interval division.

When the LoS path of any UE $l\left(l \in \mathcal{N}_{u}\right)$ is in Zone ${ }_{i}$, namely, the AoD $\left(\phi_{m}^{l}, \theta_{m}^{l}\right)$ of the path satisfies $\left|\phi_{m}^{l}-\phi_{i}\right| \leq \Psi_{h}^{i} / 2$, we can obtain the group number where the path is located immediately, with ${ }^{2}$

$$
k=\left\lfloor\left(\theta_{m}^{l}-\theta_{i}+0.5 \Theta_{h}^{i}\right) / \Theta_{h}^{i}\right\rfloor .
$$

To facilitate the representation, let $z_{i k}=k-K_{i}^{\mathrm{min}}+1$, $\forall k \in\left\{K_{i}^{\min }, K_{i}^{\min }+1, K_{i}^{\min }+2, \ldots, K_{i}^{\max }\right\}$ such that $z_{i k} \in \mathcal{Z}_{i}=\left\{1,2, \ldots, Z_{i}\right\}$, where $Z_{i}=K_{i}^{\max }-K_{i}^{\min }+1$.

\footnotetext{
${ }^{2}$ Similar method of group numbering is used in Sect. 3.1 of [7], however, there is an error in the description of ' $\theta_{s}^{t} \in\left[\theta_{\text {new }}^{t}-i \Theta_{h} / 2, \theta_{\text {new }}^{t}+i \Theta_{h} / 2\right]$ ' in step 5 of its corresponding algorithm 1 , which should be corrected to ' $\theta_{s}^{t} \in\left[\theta_{\text {new }}^{t}+(i-0.5) \Theta_{h}, \theta_{\text {new }}^{t}+(i+0.5) \Theta_{h}\right]$ '.
} 
In the downlink Los path scenario, the horizontal azimuth of all squint beams of a certain subarray is kept the same, and the subarray is grouped in the vertical elevation angle range by the vertical HPBW of the main beam only. When $\phi_{i}$ is determined, the grouping of the subarray in the vertical angle of the transmit beam can be changed by adjusting the vertical elevation angle $\theta_{i}$ of this subarray's main beam. In this case, the problem of maximizing the number of UEs served by this subarray is

$$
\begin{array}{ll}
\max _{\theta_{i}} & \left\|\mathbf{u}_{i}\right\|_{0}, \\
\text { s.t. } & {\left[\mathbf{u}_{i}\right]_{z_{i k}}=\left\|\left[\mathbf{G}_{i}\right]_{: z_{i k}}\right\|_{0}, \quad z_{i k} \in \mathcal{Z}_{i},} \\
& 0 \leq \theta_{i} \leq \pi / 2,
\end{array}
$$

where $\mathbf{G}_{i}$ is a $N_{u} \times Z_{i}$-dimensional matrix whose individual elements take values in $\{0,1\}$, and $\left[\mathbf{G}_{i}\right]_{n_{u}, z_{i k}}=1$ denotes the channel path of the UE $n_{u}$ present in the $k$-th grouping in the elevation angle division interval of this subarray. And $\mathbf{u}_{i}$ is the $Z_{i}$-dimensional indicator vector, whose $z_{i k}$-th element is $\left[\mathbf{u}_{i}\right]_{z_{i k}}=\left\|\left[\mathbf{G}_{i}\right]_{:, z_{i k}}\right\|_{0}$, indicating the number of UE channel paths existing in the corresponding angle interval $k$, which also indicates the number of UEs existing in that angle interval in a single path channel scenario. Since it is assumed that each interval can serve at most one user, in this case $\left\|\mathbf{u}_{i}\right\|_{0}$ indicates the number of UEs that can be served simultaneously by this subarray.

3.2 User grouping based on subarray collaboration: maximizing the number of UEs served The subarrays select vectors in the beamspace determined by (11) as the precoding vectors of the steering beams at the center frequency to ensure the orthogonality among multiple transmit beams, and multiplex the array response of each subarray by squint beams, thus also ensuring the orthogonality among possible co-channel squint steering beams of multi-subarray.

Since there are $N_{\text {sub }}$ subarrays at the BS side, to maximize the number of UEs served in the system, it is necessary to find $N_{\text {sub }}$ zones such that the sum of the number of groups of existing users in these zones is maximized, namely

$$
\begin{aligned}
& \max _{\phi_{i}, \theta_{i}, i \in \mathcal{N}_{\text {sub }}} \sum_{i \in \mathcal{N}_{\text {sub }}}\left\|\mathbf{u}_{i}\right\|_{0}, \\
& \text { s.t. } \quad\left[\mathbf{u}_{i}\right]_{z_{i k}}=\left\|\left[\mathbf{G}_{i}\right]_{: ; z_{i k}}\right\|_{0}, i \in \mathcal{N}_{\text {sub }}, z_{i k} \in \mathcal{Z}_{i}, \\
& \left(\phi_{i}, \theta_{i}\right) \in \mathcal{B}_{\text {AoD }}, \quad i \in \mathcal{N}_{\text {sub }}, \\
& \left|\phi_{i}-\phi_{j}\right| \geq\left(\Psi_{h}^{i}+\Psi_{h}^{j}\right) / 2, i \neq j, i, j \in \mathcal{N}_{\text {sub }},
\end{aligned}
$$

where the meaning of $\left[\mathbf{u}_{i}\right]_{z_{i k}}$ in (22b) is consistent with the corresponding description in problem (21), and $\mathcal{B}_{\mathrm{AoD}}$ in (22c) is the set of the main beam directions corresponding to the steering beam vectors in the planar array beamspace, denoted as 


$$
\begin{aligned}
& \mathcal{B}_{\mathrm{AoD}}=\left\{\left(\phi_{m}^{\operatorname{card}\left(\mathcal{M}_{y}\right) i+l}, \theta_{m}^{\operatorname{card}\left(\mathcal{M}_{y}\right) i+l}\right):\right. \\
& \beta_{x}^{i}+\beta_{y}^{l} \leq \sqrt{2} \pi \sin \theta_{m}^{\max }, \theta_{m}^{\operatorname{card}\left(\mathcal{M}_{y}\right) i+l} \leq \theta_{m}^{\max } \\
& \left.\beta_{x}^{i}=2 i \pi / M_{x}, \beta_{y}^{l}=2 l \pi / M_{y}, i \in \mathcal{M}_{x}, l \in \mathcal{M}_{y}\right\}
\end{aligned}
$$

And (22d) makes the set of UEs served by each subarray through squint beams disjoint by restricting the horizontal angular spacing of the main beams of any two subarrays $i$ and $j$ to be greater than the horizontal HPBW of these two beams, that is, $\left|\phi_{i}-\phi_{j}\right|>\left(\Psi_{h}^{i}+\Psi_{h}^{j}\right) / 2$.

\subsection{User grouping based on subarray collaboration: a suboptimal algorithm implementation}

Algorithm 1 provides a suboptimal algorithmic implementation of the optimal squint user grouping problem based on the beamspace codebook of the subarray shown in (22).

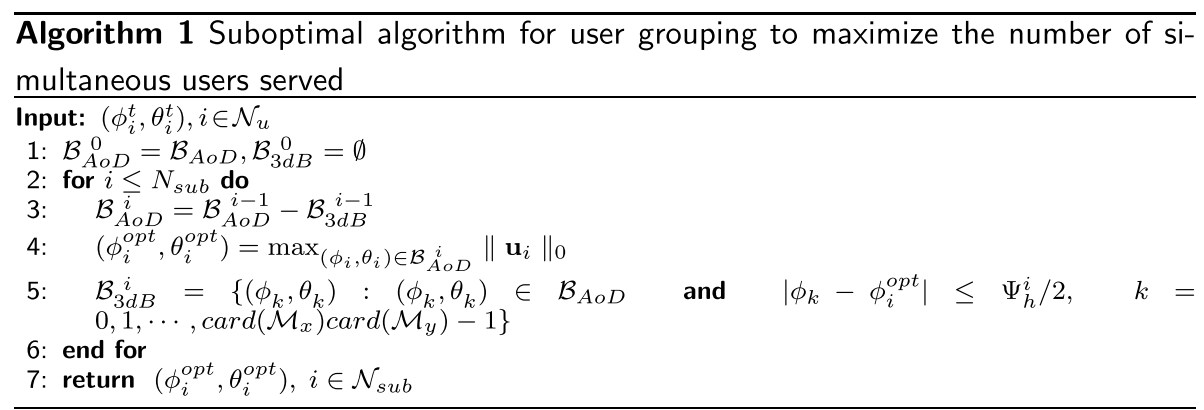

In the step 2, the main direction of the transmitting beam is selected in the beamspace for each subarray in turn by iteration.

In the step 3, the beam vectors within the HPBW of the horizontal azimuth of the steering beam already selected by the previous subarrays are excluded from the beamspace in each iteration, and this new beamspace is taken as the set of selectable beam vectors.

In the step 4, firstly, the number of users that can be served in each beam direction in the beamspace of this subarray is found, and one of the beam directions with the largest number of serving users is picked randomly as the main direction at the center frequency of the subarray, obtaining the phase differences $\beta_{x}^{i}$ and $\beta_{y}^{i}$ of adjacent elements along the $x$-axis and $y$-axis, respectively. After that, the users to be served are selected in the horizontal azimuth HPBW range of this beam, and these users are grouped in the elevation direction by the squint grouping method in the Sect. 3.1. If more than one user exists in a group, the user with the strongest channel path coefficient is selected for service. The elevation angle of the squint main beam can be chosen to coincide with the elevation angle of the channel path AoD of the serving users by selecting the appropriate frequency. When both $\beta_{x}^{i}$ and $\beta_{y}^{i}$ are of non-zero value, its operating frequency can be obtained according to Eq. (31) in [6] as 


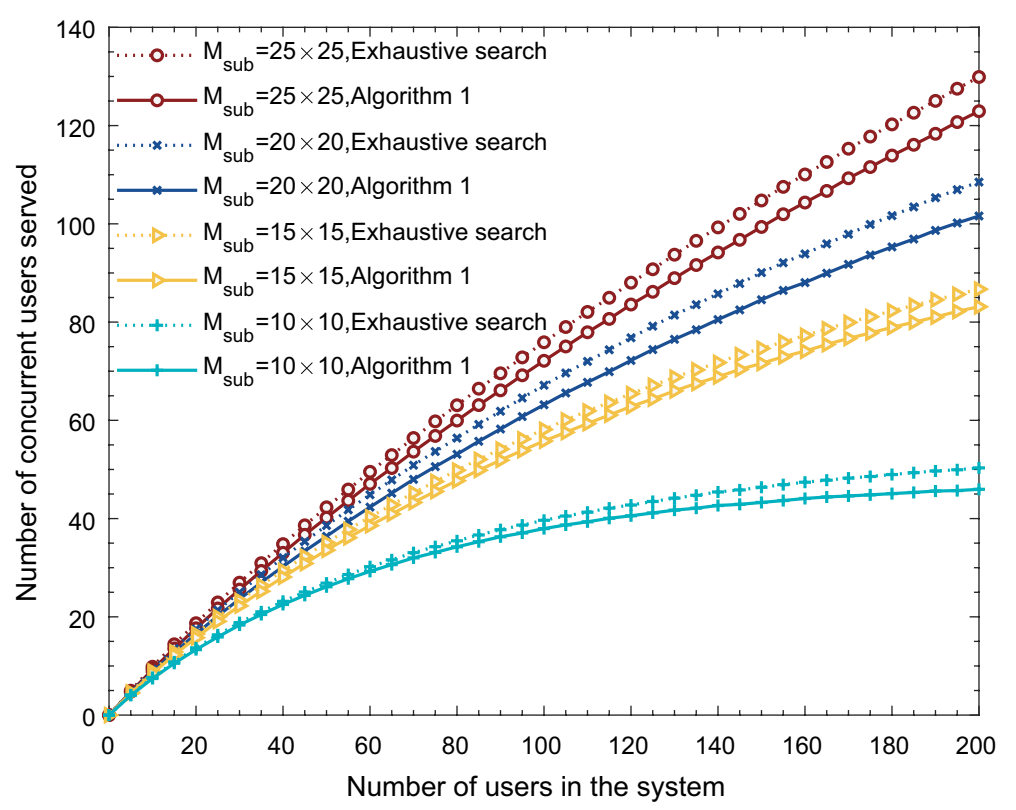

Fig. 2 Comparison of the number of simultaneous users served in the system

$$
f_{k}=\frac{f_{c} \sqrt{\beta_{x}^{i^{2}}+\beta_{y}^{i^{2}}}}{\pi \sin \left(\theta_{m}^{k}\right)} .
$$

In the step 5, the set of beam directions within the horizontal azimuthal HPBW of the selected beam in the beamspace is obtained, in preparation for finding the set of available beam directions in the beamspace of subarray in the step 2 of the next iteration. Once this set is empty, subsequent subarrays are not activated after this iteration.

\section{Results and discussion}

The simulation in this section is based on a single-cell downlink wideband scenario with the center operating frequency at $45 \mathrm{GHz}$ and the frequency range of the squint beam from 22.5 to $67.5 \mathrm{GHz}$. The BS side is equipped with 20 subarrays whose maximum elevation angle at the center frequency is set to $\pi / 3$. There are $N_{\text {sub }}$ single antenna UEs in the system, and their respective LoS paths all exist with the horizontal azimuth and vertical elevation angles obeying uniform distribution in the range of $[0, \pi / 2]$. The distance between each UE and the BS is randomly taken in the range of 10 to 100 meters, and the phase $\psi_{i}$ of the channel coefficients is randomly taken in the range of $[0,2 \pi]$. Both the user signal and the noise at the receiver side obey $\mathcal{C N}(0,1)$ distribution. Since it is a LoS scenario, the $\frac{\rho_{i}}{\sigma_{i}}$ of each user is set to be kept at $40 \mathrm{~dB}$. Finally, all results are reported for averaged over 5000 random channel realizations. Unless otherwise mentioned, these parameters are used for the experiments.

Figures 2, 3, and 4 compare the performance of the number of users served, the number of subarrays used, and the system sum rate performance of Algorithm 1 with the exhaustive method for systems with the subarray antenna configurations of $10 \times 10$, $15 \times 15,20 \times 20$, and $25 \times 25$, respectively. 


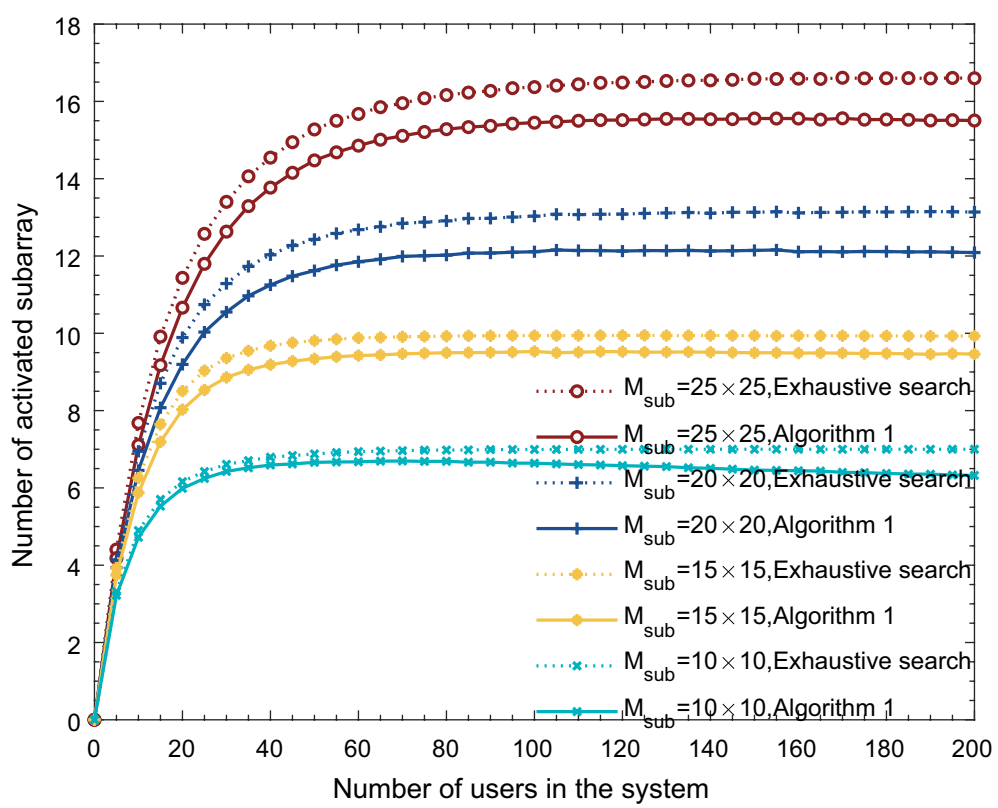

Fig. 3 Comparison of the number of active subarrays in the system

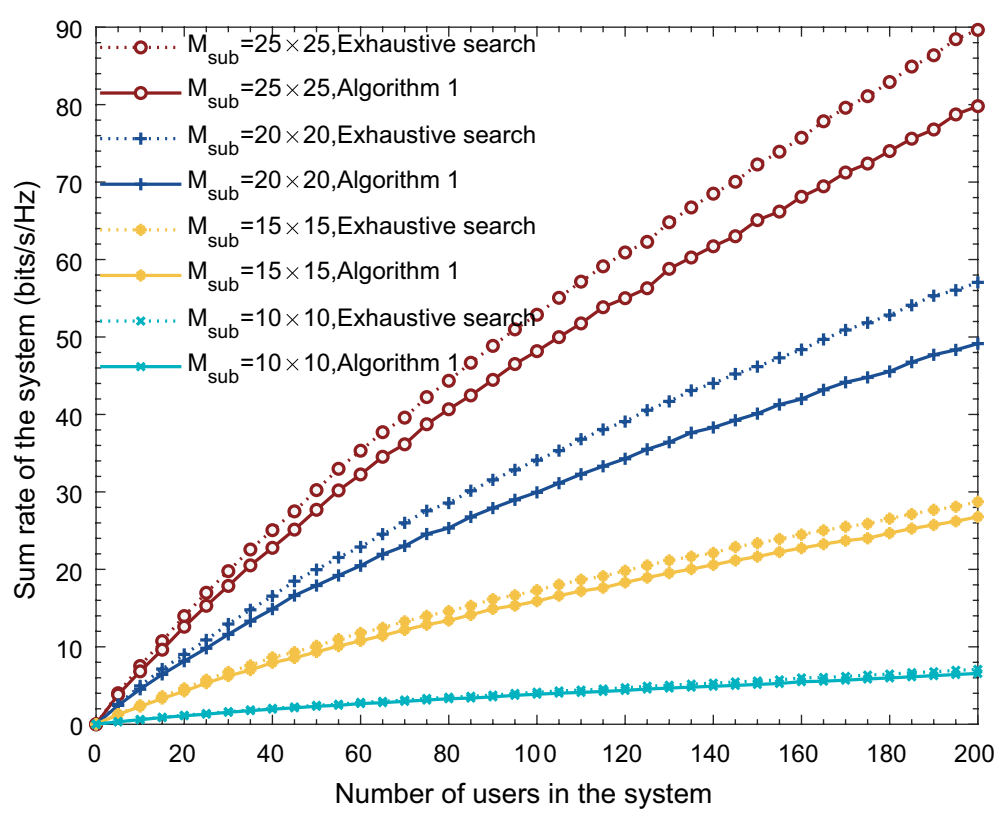

Fig. 4 Comparison of sum rates in the system

In Fig. 2 for various antenna configurations, the performance of the number of UEs served by Algorithm 1 is closer to the optimal number of UEs served by the system obtained by the exhaustive method. In addition, as the total number of users in the system increases, the configuration with fewer subarray antennas tends to be smoother first, which is caused by the smaller number of subarray antennas with a 
wider HPBW in the elevation direction of the main beam resulting in a smaller number of elevation groups.

In Fig. 3, the number of subarrays collaborating in both Algorithm 1 and the exhaustive method under the same subarray configuration gradually tends to smooth out with the increase of the number of UEs in the system, and the number of subarrays working concurrently is less when the number of subarray antennas tends to smooth out in the configuration with fewer subarrays, which is because the horizontal HPBW of the main beam is wider when the number of subarray antennas is smaller. Besides, the number of working subarrays of Algorithm 1 is slightly lower than that of the exhaustive method, with no more than one subarray in most scenarios. Moreover, it is worth noting that the curve of Algorithm 1 has a slight decreasing trend after leveling off as the number of UEs in the system increases when the subarray configuration is $10 \times 10$. The reason for this phenomenon is that, on the one hand, the number of beamspace zones is smaller when the number of subarray antennas is small, and the selection order of different zones of Algorithm 1 has a greater impact on the number of activated subarray due to the larger horizontal HPBW range, and on the other hand, as the number of UEs in the system increases, there are more UEs in the squint groups of each beamspace zone, and since Algorithm 1 first selects beams randomly in the set of optimal zones, which amplifies the effect of the randomness in the selection of zones by subarrays.

In Fig. 4, the sum rate performance of both Algorithm 1 and the exhaustive method increases with the number of UEs in the system, and the sum rate increases with more antennas in the subarray. Also, the sum rate performance of the system with Algorithm 1 is lower than that with the exhaustive method and stays within a certain interval, which is smaller with fewer subarray antennas.

Since the algorithmic complexity of the exhaustive method is $\mathcal{O}\left(\operatorname{card}(\mathbb{B})^{N_{\text {sub }}}\right)$, and Algorithm 1 with a complexity of only $\mathcal{O}\left(N_{\text {sub }}\right)$ obtains a performance closer to it, and therefore is a comparatively desirable suboptimal algorithm.

\section{Conclusion}

To further enhance the number of UEs served by the wideband planar array squint multi-beam at the BS end and to expand the departure angle range of the serviceable user path, a beamspace squint user grouping scheme based on the collaboration of subarrays is investigated in the LoS multi-user downlink scenario with multi-subarray at the BS end. First, we give the beamspace partitioning at the center frequency and prove that the uniform quantization of the wideband ideal phase shifter and the precoding scheme based on the beamspace codebook are compatible. Then, we give the way of squint user grouping in the elevation direction, propose the problem of maximizing the number of served squint users in the system based on the beamspace codebook of the collaborative subarrays, and give a suboptimal algorithm by gradually shrinking the set of serviceable beams in the beamspace codebook. Finally, we verify the feasibility of the proposed user grouping method with multi-subarray collaboration and the performance of the proposed suboptimal algorithm by simulation experiments. It is worth mentioning that the squint user grouping method and the suboptimal algorithm proposed in this paper are also very suitable for systems equipped with fully connected hybrid analog-digital transceivers. 


\section{Abbreviations}

mmWave: Millimeter-wave; RF: Radio frequency; UE: User equipment; BS: Base station; IoV: Internet of Vehicles; AoD: Angle-of-departure; HPBW: Half-power beamwidth.

\section{Acknowledgements}

Not applicable.

\section{Authors' contributions}

XP contributed with the research idea, methods, simulations, and was a major contributor in writing the manuscript. CL and LY further examined the manuscript and corrected it. All authors read and approved the final manuscript.

\section{Funding}

This work was supported in part by the National Key R\&D Program of China under Grant 2020YFB1804901, in part by the National Natural Science Foundation of China under Grants U1936201 and 61971128.

Availability of data and materials

Data sharing not applicable to this article as no datasets were generated or analysed during the current study.

\section{Declarations}

Competing interests

The authors declare that they have no competing interests.

Received: 18 November 2021 Accepted: 27 December 2021

Published online: 15 January 2022

\section{References}

1. W. Hong, Z.H. Jiang, C. Yu, J. Zhou, P. Chen, Z. Yu, H. Zhang, B. Yang, X. Pang, M. Jiang et al., Multibeam antenna technologies for $5 G$ wireless communications. IEEE Trans. Antennas Propag. 65(12), 6231-6249 (2017)

2. Y.J. Guo, M. Ansari, R.W. Ziolkowski, N.J.G. Fonseca, Quasi-optical multi-beam antenna technologies for B5G and 6G mmWave and THz networks: a review. IEEE Open J. Antennas Propag. 2, 807-830 (2021). https://doi.org/10.1109/ OJAP.2021.3093622

3. Y. Aslan, J. Puskely, A. Roederer, A. Yarovoy, Active multiport subarrays for 5G communications, in 2019 IEEE-APS Topical Conference on Antennas and Propagation in Wireless Communications (APWC) (IEEE, 2019), pp. 298-303

4. O.E. Ayach, S. Rajagopal, S. Abu-Surra, Z. Pi, R.W. Heath, Spatially sparse precoding in millimeter wave MIMO systems. IEEE Trans. Wirel. Commun. 13(3), 1499-1513 (2014). https://doi.org/10.1109/TWC.2014.011714.130846

5. R.J. Mailloux, Phased Array Antenna Handbook (Artech House, London, 2017)

6. X. Pan, C. Li, M. Hua, W. Yan, L. Yang, Compact multi-wideband array for millimeter-wave communications using squint beams. IEEE Access 8, 183146-183164 (2020). https://doi.org/10.1109/ACCESS.2020.3027835

7. X. Pan, L. Yang, Downlink multi-user algorithms for millimeter-wave wideband linear arrays on PD-NOMA-based squint steering beams. EURASIP J. Adv. Signal Process. 2021(1), 1-26 (2021)

8. A. Sayeed, N. Behdad, Continuous aperture phased MIMO: basic theory and applications, in 2010 48th Annual Allerton Conference on Communication, Control, and Computing (Allerton) (IEEE, 2010), pp. 1196-1203

9. A. Sayeed, J. Brady, Beamspace MIMO for high-dimensional multiuser communication at millimeter-wave frequencies, in 2013 IEEE Global Communications Conference (GLOBECOM) (IEEE, 2013), pp. 3679-3684

10. X. Liu, X. Zhang, Noma-based resource allocation for cluster-based cognitive industrial internet of things. IEEE Trans. Ind. Inform. 16(8), 5379-5388 (2020). https://doi.org/10.1109/TII.2019.2947435

11. X. Liu, X.B. Zhai, W. Lu, C. Wu, QoS-guarantee resource allocation for multibeam satellite industrial internet of things with NOMA. IEEE Trans. Ind. Inform. 17(3), 2052-2061 (2021). https://doi.org/10.1109/TII.2019.2951728

12. X. Liu, X. Zhang, Rate and energy efficiency improvements for 5G-based loT with simultaneous transfer. IEEE Internet Things J. 6(4), 5971-5980 (2019). https://doi.org/10.1109/JOT.2018.2863267

13. X. Liu, X. Zhang, M. Jia, L. Fan, W. Lu, X. Zhai, 5G-based green broadband communication system design with simultaneous wireless information and power transfer. Phys. Commun. 28, 130-137 (2018)

14. F. Li, K.-Y. Lam, X. Liu, J. Wang, K. Zhao, L. Wang, Joint pricing and power allocation for multibeam satellite systems with dynamic game model. IEEE Trans. Veh. Technol. 67(3), 2398-2408 (2018). https://doi.org/10.1109/TVT.2017. 2771770

15. J. Brady, A. Sayeed, Beamspace MU-MIMO for high-density gigabit small cell access at millimeter-wave frequencies, in 2014 IEEE 15th International Workshop on Signal Processing Advances in Wireless Communications (SPAWC) (IEEE, 2014), pp. 80-84

16. H.T. Friis, A note on a simple transmission formula. Proc. IRE 34(5), 254-256 (1946)

17. C.A. Balanis, Antenna Theory: Analysis and Design (Wiley, Hoboken, 2016)

\section{Publisher's Note}

Springer Nature remains neutral with regard to jurisdictional claims in published maps and institutional affiliations. 